\title{
A Case of Neuroendocrine Malignant Tumor with Capsule Retention Diagnosed by Double-Balloon Enteroscopy
}

\author{
Jie Chen ${ }^{\mathrm{a}}$ Ling Zhang ${ }^{\mathrm{a}}$ Wenjun Zhang ${ }^{\mathrm{a}}$ \\ Weiqiang Zheng ${ }^{\mathrm{b}}$ Duowu Zou ${ }^{\mathrm{a}}$ Zhaoshen $\mathrm{Li}^{\mathrm{a}}$ \\ Departments of a Gastroenterology and bPathology, Changhai Hospital, \\ The Second Military Medical University, Shanghai, China
}

\section{Key Words}

Neuroendocrine malignant tumor - Capsule - Retention - Double-balloon enteroscopy

\begin{abstract}
The diagnosis of neuroendocrine malignant tumor in the small bowel is difficult [J Oncol 2008;2008:212067]. It is usually found intraoperatively during intestinal resection and confirmed by subsequent histological examination. We reported a case of jejunum neoplasm which was misdiagnosed as Crohn's disease by capsule endoscopy. Capsule endoscopy was stuck in the jejunum stricture and was removed with a snare under double-balloon enteroscopy. The pathology of the biopsy was jejunum small cell malignant carcinoma tending to neuroendocrine malignant tumor. The immunohistochemical result revealed that CD99 and Ki-67 were positive. The patient underwent intestinal resection and the diagnosis of neuroendocrine malignant tumor was confirmed.
\end{abstract}

\section{Introduction}

The incidence of neuroendocrine tumors is increasing (approximately $6 \%$ per year), but clinical presentation is nonspecific, resulting in delays in diagnosis (5-7 years; approximately $70 \%$ have metastases) $[1,2]$. Neuroendocrine tumors usually exist at the lung, stomach and colon, but the small bowel is a rare location. We present an unusual case of small bowel neuroendocrine malignant tumor diagnosed by double-balloon enteroscopy (DBE). The jejunum neoplasm was misdiagnosed as Crohn's disease by capsule endoscopy. The capsule was stuck in the jejunum stricture and was removed with a snare under DBE. 


\section{Case Report}

A 36-year-old man with weight loss presented with a 1-year history of distention, abdominal pain and macies. Gastroscopy, colonoscopy and magnetic resonance imaging of the abdomen showed no positive findings. Capsule endoscopy found segmental distribution of ulceration, erosion and nodal hyperplasia in the proximate jejunum consistent with Crohn's disease (fig. 1a). Neither the doctor nor the patient remembered finding the capsule in the stool. The patient came to our hospital for further evaluation. Fourteen days after the capsule endoscopy examination we inserted a DBE orally. When the scope passed $20 \mathrm{~cm}$ from the Treitz ligament we found jejunum erosion, nodal hyperplasia, stenosis (fig. 1b) and a capsule there. We grasped the capsule with a snare and pulled it out (fig. 1c). The DBE was inserted again for biopsy, and some specimen were retrieved. The next day, barium examination revealed that the stenosis was near to the Treitz ligament (fig. 1d).

After six days, the pathology (fig. 2) was jejunum small cell malignant carcinoma tending to neuroendocrine malignant tumor. Immunohistochemical examination showed that CD99 and Ki-67 were positive (fig. 3 ). After consultation with a senior general surgeon, the patient was scheduled to undergo surgery. The operation went smoothly and the diagnosis of neuroendocrine malignant tumor was confirmed.

\section{Discussion}

DBE can be used to confirm capsule endoscopy findings. As we know, capsule retention is one of the most common complications of capsule endoscopy examination [3]. Capsule retention is defined as the presence of the capsule endoscopy in the digestive tract for a minimum of 2 weeks after ingestion or the capsule being retained in the bowel lumen indefinitely unless a targeted medical, endoscopic, or surgical intervention is initiated [4]. Small bowel stenosis often leads to capsule retention.

In this patient, we shared an examination process of a rare case of neuroendocrine malignant tumor in the small bowel. As the capsule endoscopy was stuck in the narrow parts, the lesion images from different angles were shot at different times, which led us to mistake for multiple lesions and to make a misdiagnosis of Crohn's disease. If we had adopted the therapeutic regimen in accordance to the diagnosis of Crohn's disease, maybe the patient would have lost the chance of operation. At the same time, we introduced the skills that the application of DBE could be used in the removal of capsule retention. 
Fig. 1. a Erosion and bleeding seen in the capsule endoscopy image. $\mathbf{b}$ The jejunum erosion, nodal hyperplasia and stenosis seen in the DBE image. c Capsule endoscope retained at the jejunum stenosis and pulled out with a snare. $\mathbf{d}$ Jejunum stenosis as seen in abdominal barium meal X-ray film.
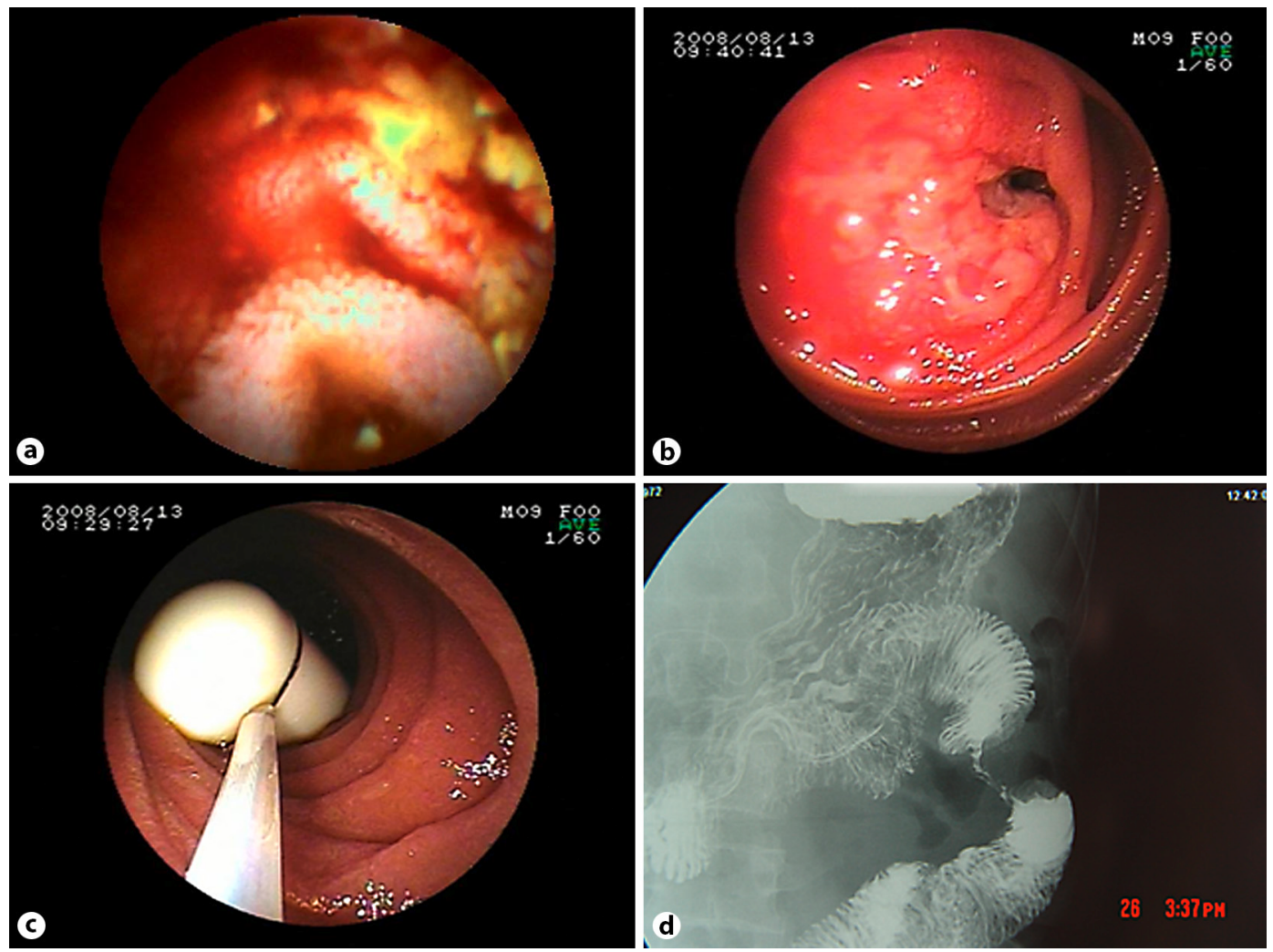

Fig. 2. The results of pathology. a $40 \times$ original magnification. b $100 \times$ original magnification.

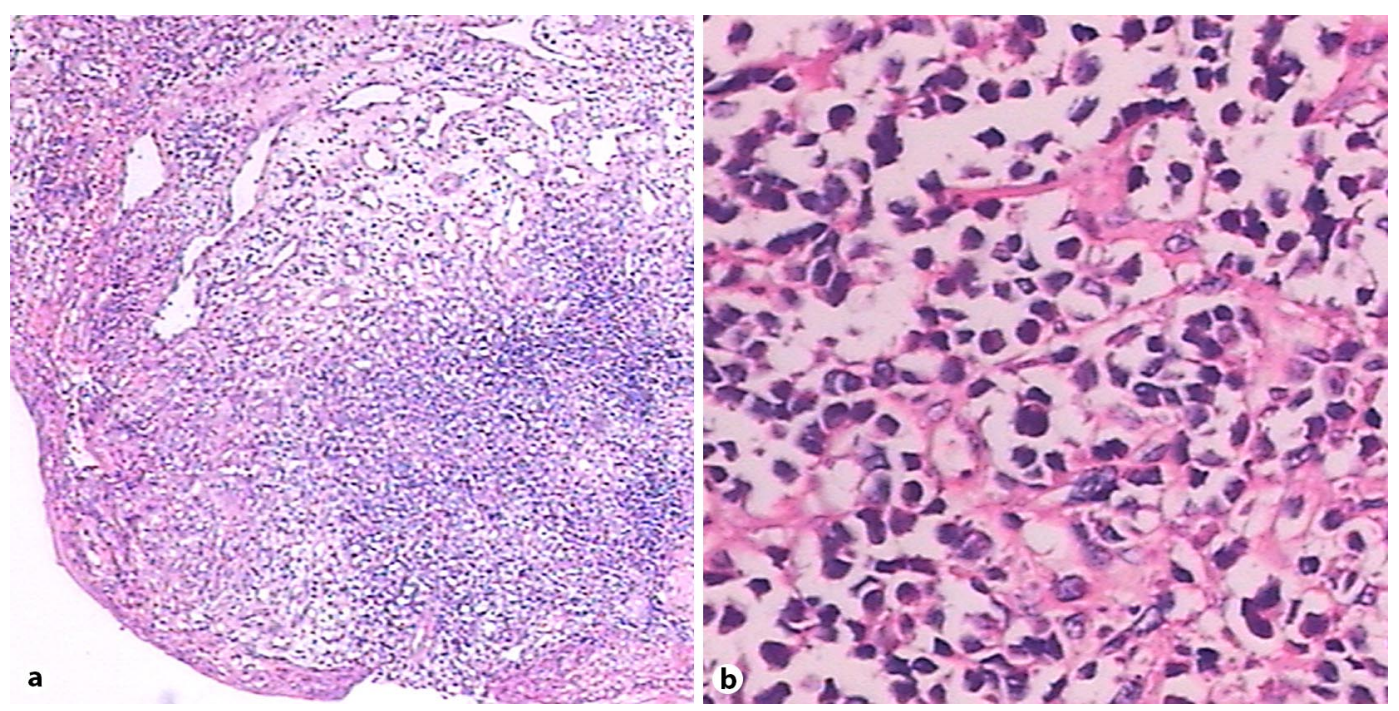




\begin{tabular}{r|l|l|l}
$\begin{array}{r}\text { Case Reports in } \\
\text { Gastroenterology }\end{array}$ & $\begin{array}{l}\text { Case Rep Gastroenterol 2010;4:52-56 } \\
\text { D01: 10.1159/000264644 }\end{array}$ & Published online: February 6, 2010 & $\begin{array}{l}\text { 2010 S. Karger AG, Basel } \\
\text { ISSN 1662-0631 } \\
\text { www.karger.com/crg }\end{array}$ \\
\hline
\end{tabular}

Fig. 3. The results of immunohistochemistry. a Positive staining of CD99. b Positive staining of Ki-67.

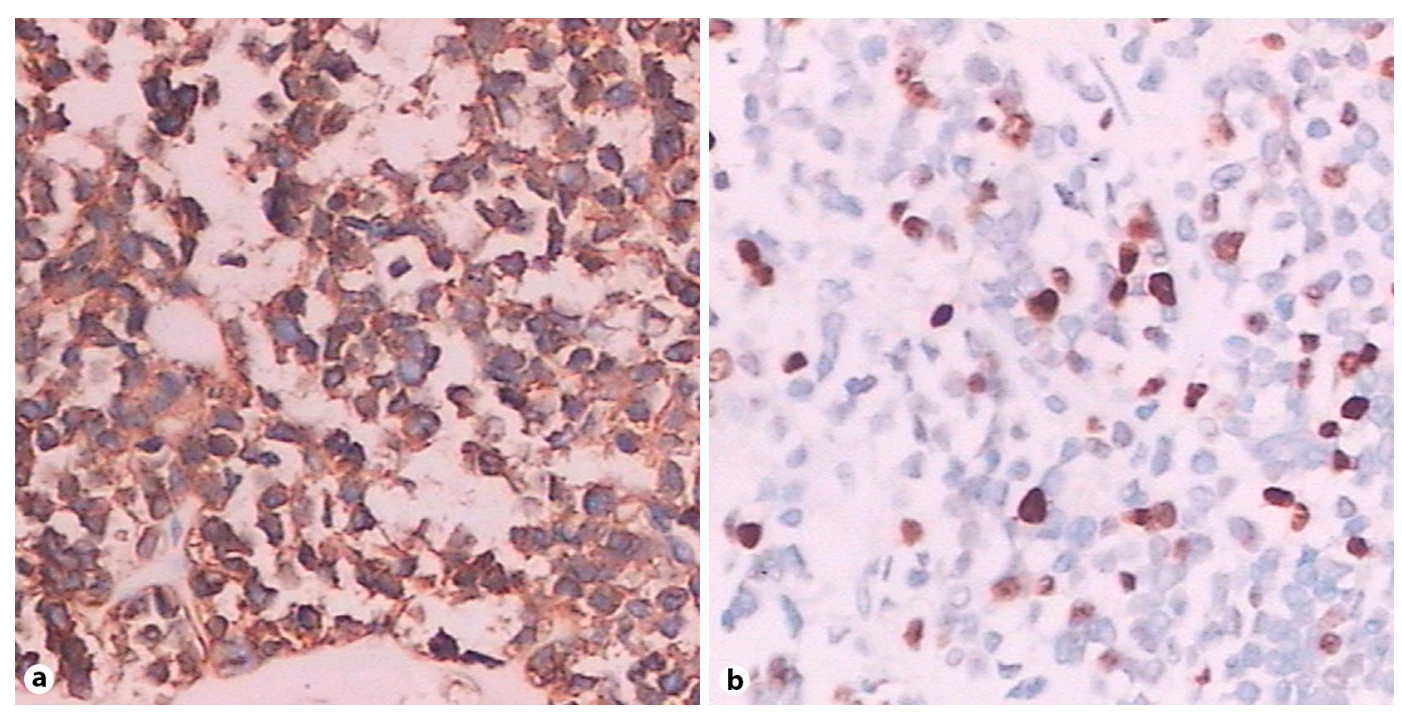




\section{References}

1 Farhat MH, Shamseddine AI, Barada KA: Small bowel tumors: clinical presentation, prognosis, and outcome in 33 patients in a tertiary care center. J Oncol 2008;2008:212067.

-2 Modlin IM, Gustafsson BI, Drozdov I, et al: Principal component analysis, hierarchical clustering, and decision tree assessment of plasma mRNA and hormone levels as an early detection strategy for small intestinal neuroendocrine (carcinoid) tumors. Ann Surg Oncol 2009;16:487-498.

-3 Cave D, Legnani P, de Franchis R, et al: ICCE consensus for capsule retention. Endoscopy 2005;37:1065-1067.

-4 Li F, Gurudu SR, De Petris G, et al: Retention of the capsule endoscope: a single-center experience of 1,000 capsule endoscopy procedures. Gastrointest Endosc 2008;68:174-180. 